\title{
Radiotherapy for brain metastases from small-cell lung cancer in distinct clinical indications and scenarios
}

\author{
Lucyna Kepka ${ }^{1}$, Joanna Socha ${ }^{1}$, Beata Sas-Korczynska ${ }^{1,2}$ \\ ${ }^{1}$ Department of Radiotherapy, Military Institute of Medicine, Warsaw, Poland; ${ }^{2}$ Institute of Medical Sciences, Medical College of Rzeszow \\ University, Rzeszow, Poland \\ Contributions: (I) Conception and design: L Kepka; (II) Administrative support: None; (III) Provision of study materials or patients: None; (IV) \\ Collection and assembly of data: None; (V) Data analysis and interpretation: None; (VI) Manuscript writing: All authors; (VII) Final approval of \\ manuscript: All authors. \\ Correspondence to: Lucyna Kepka. Department of Radiotherapy, Military Institute of Medicine, Warsaw, Poland. Email: 1kepka@wim.mil.pl.
}

\begin{abstract}
Management of brain metastases (BM) from small-cell lung cancer (SCLC) is complex and not supported by a strong evidence from prospective clinical trials. Owing to the different clinical and pathological characteristics of SCLC, patients with this histology were not included in the prospective studies on the value of whole-brain radiotherapy (WBRT) and local surgical or ablative radiation treatment like stereotactic radiosurgery (SRS). Chemotherapy also represents a major part of the armamentarium against BM from SCLC due to the well-recognized chemoresponsiveness of this cancer and the frequent presentation of BM with extracranial progression. WBRT in combination with chemotherapy has long been a standard approach in this setting. However, data on the neurocognitive toxicity and the lack of documented impact on overall survival of WBRT in the management of BM from other solid tumors, as well as the increasing availability of the stereotactic radiotherapy technologies, has led to the increasing use of SRS with omission of WBRT also in SCLC. In the current review the use of different modalities of radiotherapy and ways of combining radiotherapy with chemotherapy for BM from SCLC will be presented for distinct clinical situations: presentation of $\mathrm{BM}$ synchronous with primary, metachronous presentation of $\mathrm{BM}$-without previous prophylactic cranial irradiation (PCI) vs. after PCI, and asymptomatic BM found at the staging before PCI.
\end{abstract}

Keywords: Small-cell lung cancer (SCLC); brain metastases (BM); whole-brain radiotherapy (WBRT); stereotactic radiosurgery (SRS)

Submitted Sep 06, 2019. Accepted for publication Oct 14, 2019.

doi: $10.21037 /$ jtd.2019.10.51

View this article at: http://dx.doi.org/10.21037/jtd.2019.10.51

Small-cell lung cancer (SCLC) is a rapidly progressing disease with a predilection for early metastasizing. The most common site of metastases is the brain; about $40-50 \%$ of patients develop brain metastases (BM) within 2 years from diagnosis (1). Radiotherapy plays a crucial role in the management of BM from SCLC. However, there are a number of distinct biological and clinical characteristics of SCLC that preclude the direct incorporation of the results of the studies on the treatment of $\mathrm{BM}$ from other solid tumors to the management of BM from SCLC. These are: the high aggressiveness of the disease with rapid micro- and macro-dissemination to the brain, the rare occurrence without the presence of extracranial disease, chemo-sensitivity that incites the use of systemic treatment, the use of prophylactic cranial irradiation (PCI) as a part of the standard strategy for patients without BM, and a dismal disease course. The historical treatment of BM from SCLC was whole-brain radiotherapy (WBRT) alone or combined with chemotherapy (CHT). The outcome of such strategies was poor; median overall survival (OS) after WBRT was 3.0-4.7 months in both prospective and retrospective studies (2-5). However, a temporal trend towards a slight survival improvement in SCLC exists (6) and is also seen for patients with BM in recently published 
population-based studies. In the cohort of 13,657 patients with BM from SCLC managed with WBRT in the USA between 2004 and 2013, a median OS of 8 months was demonstrated (7). In another study, also based on the data from the USA National Cancer Database (NCDB), 5,752 patients managed between 2010 and 2014 with WBRT for BM from SCLC had a median OS of 7 months (8). Thus, contemporarily treated patients probably had a slightly better, yet still disappointingly low survival chance.

Technological advances in radiotherapy, the neurocognitive toxicity of WBRT, the lack of impact of WBRT on survival in BM from other solid tumors led to the increased use of up-front stereotactic radiosurgery (SRS) with omission of WBRT also in SCLC (7). However, a high potential of SCLC for diffuse dissemination in the central nervous system (CNS), the impact of PCI on survival in the absence of overt BM $(9,10)$, and the exclusion of SCLC patients from the trials on radiotherapy of BM (11-15) indicate that the optimal way of delivering radiotherapy (WBRT vs. SRS or both) for patients with BM from SCLC remains to be defined. In the current review we present some evidence and unresolved issues on the use of radiotherapy for patients with BM from SCLC in distinct clinical situations: (I) in newly diagnosed SCLC, (II) in asymptomatic BM, found at the staging before PCI, (III) at metachronous presentation-without previous PCI vs. after PCI. Each of these clinical situations will be also presented in the context of the suitability of the patient for the use of SRS, the role of WBRT, and the use of systemic treatment.

\section{BM in synchronous presentation with initial diagnosis of SCLC}

Brain imaging [magnetic resonance imaging (MRI) preferred over computerised tomography (CT)] is mandatory in the initial staging of SCLC, because of the frequency of BM in SCLC at presentation (about 15\%) (1). Even if other distant metastases are detected at the initial work-up, brain imaging is still recommended with a view to avoiding an early neurological deterioration from untreated $\mathrm{BM}$ and to prevent complications during CHT delivery, as well as to properly select patients for PCI $(16,17)$. The firstline treatment for such patients is CHT, due to the wellknown chemo-sensitivity of SCLC and the need to start the treatment without delay in this very aggressive cancer in view of avoiding deterioration of performance status related to the systemic disease progression.

A question remains concerning the way of introduction of brain radiotherapy in relation to $\mathrm{CHT}$ in such patients and the efficacy of CHT itself in the management of BM from SCLC. The use of CHT even for chemo-sensitive tumors has been questioned, because of the notion that the bloodbrain barrier (BBB) is impenetrable/poorly penetrated by most drugs and therefore the brain is a pharmacological sanctuary site for microscopic tumors. However, it has been shown that cytotoxic agents may achieve a good penetration into the brain, because the BBB is damaged by BM, and there are clinical data confirming it (18). In the pooled data from five studies on 64 patients with synchronous $\mathrm{BM}$, a $66 \%$ response rate (RR) was demonstrated (19). On the other side, the RR is usually lower in the brain than in the extracranial sites; in the study on 24 asymptomatic patients with BM from SCLC who received CHT with cyclophosphamide, doxorubicine, and vincristine, the RR was $27 \%$ in the brain and $73 \%$ outside the brain (20). There is only one randomized trial that compared CHT alone (teniposide) vs. teniposide + WBRT 30 Gy. In this trial, 120 patients with progression in the brain after or during firstline CHT were included. CHT alone led to a significantly shorter time to progression within the brain $(\mathrm{P}=0.005)$. $\mathrm{RR}$ were $57 \%$ and $22 \%$ for combined modality arm and CHT alone arm respectively, $\mathrm{P}<0.001$. OS was not different in both arms (3). Additionally, a Cochrane Review evaluating a role of CHT for BM from SCLC found that there was insufficient evidence to indicate a survival advantage for CHT alone (21). Recently, it was demonstrated that the addition of atezolizumab [a humanized monoclonal antiprogrammed death ligand 1 (PD-L1) antibody] to CHT (carboplatin + etoposide) in the first-line treatment of extensive-stage (ES) SCLC resulted in a significantly longer OS than CHT alone. In included patients with BM, no difference between the two groups was observed in OS or progression-free survival (PFS). However, patients with BM represented only $9 \%$ of the entire group. Thus, the firm conclusion about the role of immunotherapy in the management of BM from SCLC cannot be drawn (22). Certainly, further research is needed in this area. Nevertheless, the presented data (risk of rapid progression in the brain without the use of WBRT and lower RR in the brain) suggests that radiotherapy should be considered for asymptomatic patients after completion of $\mathrm{CHT}$ regardless of CHT response. However, we should be aware that strong evidence for such an approach is lacking. Recently, the USA NCDB-based study on 1615 elderly patients ( $\geq 75$ years) with BM from SCLC showed that WBRT did not improve OS in patients who received CHT; median OS was 5.6 and 
6.4 months with and without WBRT, respectively, $\mathrm{P}=0.43$. In patients without CHT, median OS rates were 1.9 and 1.2 months with and without WBRT, respectively, $\mathrm{P}<0.0001$ (23). These findings suggest that in a fragile population of elderly patients who are still able to receive CHT, an omission of WBRT may be considered.

In addition, there is a special case when SCLC histology is found unexpectedly in a brain tumor during a craniotomy without prior diagnosis of the lung primary of this histology. Obviously, the staging procedures in order to find the lung primary and the extracranial extensions of the disease should be launched before the therapeutic decision. When the lung primary is found, the management is not different than in the case of the unremoved $\mathrm{BM}$ diagnosed during an initial staging of SCLC, namely it is CHT followed by WBRT. SRS of the tumor bed is not yet a standard of care in SCLC, because of the increased risk of brain failure elsewhere. For this reason, the BM from SCLC were also not included in the clinical trials that compared the use of tumor bed SRS with the omission of WBRT $(14,15)$.

The above considerations were mainly dedicated to the treatment of asymptomatic or slightly symptomatic patients with BM. For symptomatic BM patients, a customary practice is to start treatment with radiation in order to improve the patient's condition before the start of CHT, because the response in the brain is more likely to occur with radiotherapy. However, such decisions should be taken jointly with a medical oncologist.

WBRT following CHT in asymptomatic patients remains the standard treatment of synchronous $B M$ in newly diagnosed SCLC. There is no strong evidence for the use of SRS in this indication. However, the increasing tendency towards the use of SRS with omission of WBRT in BM from SCLC is observed (7). This issue is discussed in more details below.

\section{Pre-PCI imaging for limited stage (LS) or ES SCLC}

Results of meta-analyses confirmed that PCI reduces BM incidence and improves OS rates in both LS and ES SCLC $(9,24)$. Taking into account the low percentage of patients with ES included in the meta-analysis $[15 \%$ of patients in Auperin et al. meta-analysis (9)], and a short OS of ES SCLC patients with practically no long-term survivors, the use of PCI for this group was more debatable (25). For a long time, PCI was recommended only for LS SCLC patients after completion of (radio) CHT. Based on the effectiveness and toxicity data, PCI at the dose of $25 \mathrm{~Gy}$ in 10 fractions has been recommended for LS SCLC patients who have a good response to CHT $(26,27)$. The EORTC phase 3 trial demonstrated that also the ES SCLC patients who responded to the first-line CHT had improved survival with the use of PCI; 1-year OS rates were $27.1 \%$ and $13.3 \%$ in the PCI and non-PCI (control) group, respectively, $\mathrm{P}=0.003$. Brain imaging was not a part of standard staging, neither at baseline, nor before PCI, unless symptoms suggestive of BM were present (10). Contrarily, in the Japanese trial that evaluated a value of PCI in ES SCLC patients, the MRI was required at baseline, before randomisation and every 3 months in the follow-up. This study confirmed that PCI reduces the risk of BM development without any survival benefit. There was even a non-significant trend for longer survival observed in patients who did not receive PCI (17).

Brain imaging has not always been a standard procedure before qualification for PCI in either LS or ES SCLC. NCCN guidelines recommend pre-PCI MRI for patients with response to initial therapy (28). Some prospective studies reported using MRI or CT scans, some did not require any imaging, and some did not mention any requirements for imaging $(29,30)$. A recent survey conducted in the USA, demonstrated that up to $96 \%$ of 309 radiation oncologists performed pre-PCI MRI (31). The European practice differs in this regard; according to the recommendations, the brain imaging is not mandatory before PCI, at baseline in elsewhere confirmed ES, or during follow-up in the absence of symptoms (32). Recently, in the survey on the practice of PCI for ES SCLC, some European experts from both the European Society for Therapeutic Radiation Oncology (ESTRO) and the International Association for the Study on Lung Cancer (IASLC) highlighted that they perform PCI for ES SCLC patients, because the restrictions in reimbursement for MRI and problems with its availability prevent them from the omission of PCI that was proved to prolong OS in one randomized trial, in which patients had suboptimal brain imaging performed (10). However, with MRI surveillance, patients could avoid brain irradiation, unnecessary in some cases (33).

\section{What evidence do we have for the value of performing MRI before qualification for PCI?}

Against such an approach, there are some indications that patients who are free of $\mathrm{BM}$ at baseline and develop BM during a first line treatment, have a particularly poor 
prognosis. The use of WBRT doses higher than usually prescribed for PCI did not reverse the poor prognosis of these patients in two case-series $(34,35)$. Thus, the routine use of the pre-PCI MRI would not be supported. In one study, patients with initial diagnosis of LS SCLC had a baseline MRI performed. Complete responders who qualified for PCI after treatment completion had a second, pre-PCI MRI; 13 out of 40 (32.5\%) patients harbored asymptomatic BM in the pre-PCI MRI. Despite higher WBRT doses, patients with pre-PCI detected BM had worse OS than those without BM in the pre-PCI MRI receiving standard PCI doses: $17 \%$ vs. $74 \%$ of 1 -year OS rate, respectively, $\mathrm{P}=0.0001$. Of note, the PCI was applied late in this study, between 4 and 10 months after diagnosis (34). Similar findings were presented at the IASLC World Conference on Lung Cancer in 2018. From 119 LS SCLC patients with a baseline brain MRI, referred for PCI after chemo-radiotherapy, 25 (21\%) had BM on pre-PCI MRI, and 23 were asymptomatic. Patients with $\mathrm{BM}$ in pre-PCI MRI had significantly shorter OS than those without. The duration of chemo-radiotherapy (in excess of 4.5 months) was the only prognostic factor for the occurrence of the prePCI BM (35). What we learn from these studies is that we should avoid unnecessarily prolonging chemo-radiotherapy for SCLC and start PCI as quickly as possible after the end of the first-line treatment. A radiobiological modeling study supports this opinion. When PCI was delayed for over 60 days, significantly higher doses were necessary for reduction of the risk of BM from SCLC, which is consistent with a fast growth rate of the untreated subclinical BM (36).

Performance of the pre-PCI MRI is supported by the results of the above mentioned Japanese randomized trial that challenged the routine use of PCI for ES SCLC and supported the use of the MRI brain surveillance and early salvage radiation for overt BM (17). A lesson taken from this trial is that BM identified early in the context of MRI surveillance (not as in the above case-series, with patients for whom the delay in the start of PCI led to the development of overt $\mathrm{BM}$ ), may be salvaged without negative impact on survival. It is also pointed out that the omission of PCI with a strict MRI surveillance may spare a subset of patients from brain irradiation and neurocognitive sequelae and the at-least-temporary worsening of a quality of life related to it; $42 \%$ of ES SCLC patients from this trial did not require brain radiation until death.

Pre-PCI MRI is also a pre-requisite for the PCI with hippocampal avoidance (HA), for the accurate delineation of the HA zone. The HA is one of the strategies used to reduce neurotoxicity in PCI and WBRT. The rationale for this approach is that the proliferating neuronal progenitor cells in the subgranular zone of the hippocampus play an essential role in memory function and the use of new radiation technologies, such as the IMRT technique, may spare this structure from the detrimental cognitive effects of radiation by minimizing a dose given to this region. Two prospective trials demonstrated a short-term (at 4-6 months) improvement of neurocognitive function with HA in WBRT for BM $(37,38)$. Some reports supported the safety of HA, demonstrating a risk of failure in the HA zone for SCLC patients to be less than 5\% $(35,39)$. In contrast, other studies reported a risk of failure of more than $10 \%$ in the HA zone with HA in PCI for SCLC $(40,41)$. The safety of such an approach remains to be confirmed by prospective trials, but in the meantime, when performing HA-WBRT for PCI or overt BM we need reliable imaging, i.e., a highquality MRI performed for HA planning according to recommendations (42).

Concluding, pre-PCI MRI is recommended, because for the patients treated in a timely and optimal way, early salvage WBRT may be not inferior to PCI, as showed by the prospective data from one trial in ES SCLC (17). Also, it may serve for radiotherapy planning purposes.

\section{Metachronous presentation of BM in SCLC}

Metachronous presentation is meant as the occurrence of BM after the first-line treatment for SCLC. The time of occurrence of $\mathrm{BM}$ in relation to the diagnosis of the primary (synchronous vs. metachronous BM) is considered as a prognostic factor for OS. Patients presenting with metachronous BM had worse OS compared with patients presenting with synchronous BM $(43,44)$. This is related to the very limited therapeutic arsenal after first-line CHT. Median survival after second-line CHT varies between 3 and 6 months in clinical trials (45). Additionally, BM in SCLC occur very rarely as a sole event, as demonstrated by the EORTC phase II trial, in which the very slow accrual of patients with brain-only metastases led to the premature closure of the study, before the required number of patients was reached (2). The extracranial disease progression is also a well-recognized adverse prognostic factor in BM, including SCLC $(46,47)$. Thus, a management of metachronous BM in SCLC represents a complex therapeutic problem. Additionally, the management becomes challenging if BM occur after previous PCI. We will discuss the treatment strategies for the metachronous 
BM with regard to the prior use, or not, of PCI.

\section{Metachronous BM that occur without prior use of PCI}

Owing to the poor prognosis of these patients and the lack of clear guidelines for the management of brain relapse in SCLC patients, the decisions are individual in each case. All the available treatment options, such as a second line CHT, radiotherapy alone, and radiotherapy combined with CHT have limited therapeutic potential. Taking into account the generally poor treatment outcome, in some more unfavorable cases, an active oncological treatment like $\mathrm{CHT}$ or any form of radiation should be weighed against supportive therapy with steroids alone.

The use of CHT in this setting is not a first-choice treatment, in contrast to the BM simultaneous to the newly diagnosed primary. It is conditioned by a number of factors, such as the site and extent of progression (brain only $v s$. brain and extracranial site with consideration of the extent of extracranial disease), previous response to $\mathrm{CHT}$, previous tolerance of CHT, the time interval from the last line of CHT, and performance status. In the Cochrane Database Syst Rev, a value of CHT for BM from SCLC based on three randomized trials including 192 patients was evaluated. No sufficient evidence to indicate a survival advantage for CHT use was found (21). Only one trial compared CHT with no CHT; 33 patients, including 28 with metachronous presentation, were randomized to WBRT alone vs. WBRT plus topotecan. No significant difference in survival was found between these two groups (48). Despite the lack of evidence that CHT improves brain tumor control and OS in these patients, CHT is given especially if there is also an extracranial disease progression, patients are in good performance status and are able to tolerate $\mathrm{CHT}$, previous response and tolerance of CHT were good enough, and also if the time interval from the last CHT is sufficiently long and/or available CHT options exist. This is based on the recognized chemo-responsiveness of SCLC.

Although a high RR in the brain after CHT for SCLC is recognized (19), both prospective and retrospective data show improved PFS and brain control with the addition of WBRT to CHT $(3,49)$. WBRT is usually given sequentially to CHT, unless the patient has bothersome symptoms related to the brain progression. In one small prospective study on 39 patients, sequential and concomitant (radio) CHT schedules (teniposide plus cisplatin with WBRT) were compared. No difference in OS and RR for either combination was demonstrated. The concomitant arm was revealed to be more toxic (50). The use of WBRT in $\mathrm{BM}$ from SCLC is not based on the results of randomized trials. There is also a concern about the neurotoxicity of such an approach. As mentioned above, in the populationbased study including 1,615 patients older than 74 years, the addition of WBRT to CHT did not improve OS. Thus, it cannot be excluded that asymptomatic patients may be treated with CHT alone. This approach may be preferential in fragile populations, susceptible to neurotoxicity—such as, for example, elderly patients.

Patients with BM in RTOG RPA (the Radiation Therapy Oncology Group, Recursive Partitional Analysis) class 3, that is, with poor performance status [Karnofsky performance status (KPS) <70] had a median OS about two months $(4,5,46)$. With such a short survival, the benefit of any active oncological treatment, including WBRT is doubtful. In 538 patients with BM from NSCLC unsuitable for resection or SRS, who were randomly assigned to WBRT and best supportive care (steroids) or best supportive care alone, the WBRT did not improve OS, quality of life, and reduction of the dose of steroids (51). One prospective trial that also included patients with SCLC histology aimed to determine whether WBRT had any benefit in terms of symptom palliation in 91 patients with KPS $<70$. All patients received WBRT and were asked to complete a questionnaire about their symptoms before and 1 month after WBRT. One month after WBRT, $53 \%$ of patients from this group had died or were not able to respond to the survey questions because of further deterioration of performance and/or neurological status. In the remaining $47 \%$ of patients, the intensity of symptoms of the disease significantly increased after WBRT (52). These results challenge the value of WBRT; steroids only are a reasonable option that should be proposed for such patients.

\section{Metachronous BM that occur after prior use of PCI}

PCI reduces the incidence of BM by about $50 \%$, however the risk of $\mathrm{BM}$ is not eliminated with the PCI use. The extracranial disease progression, which is a frequent event in LS SCLC and practically inevitable in ES SCLC, becomes a source of subsequent seeding into the CNS and this pattern of progression cannot be prevented by the PCI use. The results of meta-analysis and prospective trials indicate that the risk of brain relapse after PCI varies between 15 and $33 \%$, and in most studies, it is closer to about $30 \%$ than $15 \%(9,10,17,24)$. The 3 -year BM rate after PCI was $33 \%$ 
vs. $59 \%$ without PCI in a meta-analysis of seven randomized trials that compared treatment with and without PCI in LS SCLC and ES SCLC (9). In the EORTC trial on PCI in ES SCLC, the 1-year risk of symptomatic BM after PCI was $15 \%$ vs. $40 \%$ without PCI (10). In the trial that compared treatment with and without PCI with staging and strict surveillance with brain MRI in ES SCLC patients only, the 1-year (mainly asymptomatic) BM rate was as high as 33\% with PCI vs. 59\% in patients without PCI. This high rate of detection of BM seen in this study was mainly attributable to the strict surveillance with brain MRI performed every 3 months (17).

Re-irradiation of the brain that has previously received about 30 Gy with PCI is challenging because of the risk of neurotoxicity. The incidence of cognitive decline is volume dependent, especially when using large doses per fraction as in palliative settings (53). SRS appears a very appealing strategy in the previously irradiated region. The increased availability of SRS and improvements in the technology make this technique easily accessible. SRS is increasingly employed even for patients with multiple BM. It was demonstrated in a large randomized trial on 1,194 patients that SRS without WBRT in patients with 5-10 BM from solid tumors including SCLC was not inferior in terms of OS to that in patients with 2-4 BM (54). However, the possibility of using SRS depends not only on the number of BM but, most of all, of the volume of respective lesions and the total volume of the BM. The above-mentioned study that demonstrated non-inferiority of the outcome of SRS for 5-10 BM compared to 2-4 BM enrolled patients with one to ten $B M$ with a maximum diameter of the largest tumor $<3 \mathrm{~cm}$, and total volume of all $\mathrm{BM} \leq 15 \mathrm{~mL}$. Even if in one study, the criteria of inclusion in the SRS were extended to $\mathrm{BM}$ with the largest diameter of $5 \mathrm{~cm}$ and a maximum number of 10 , still $60 \%$ of 32 patients who experienced recurrence within the brain after PCI were unsuitable for SRS (55). Thus, a question arises about safety of using WBRT in such patients. It is reasonable to presume that their short life expectancy counted in weeks or months at best makes a risk of late neurotoxicity of WBRT unlikely, though there are very limited data on this. Bernhardt et al. (56) reported on 76 patients reirradiated after a PCI with a dose of $30 \mathrm{~Gy}$ in 15 fractions and with a median time between PCI and reirradiation of 14 months (range, 4-42 months). Repeat WBRT was given to 66 (88\%) of them with the doses of 20-30 Gy in 10-15 fractions. Median OS after repeat WBRT was 3 months (range: 0-12 months); about $40 \%$ of symptomatic patients improved after reirradiation. Notably, no serious, grade $>2$ toxicity was observed in these patients. These results support our assumption that WBRT with moderate doses may be beneficial for these many patients who are not candidates for SRS after prior PCI.

However, for patients suitable for SRS, the minimal invasiveness and ease of the use of SRS make the SRS a preferred salvage method after prior PCI for patients with a better prognosis, i.e., life expectancy $>3$ months. Thirteen patients with one to four $\mathrm{BM}$ from the above-mentioned series of 76 patients reirradiated after PCI received SRS with 18-24 Gy. Their median OS was 5 months; data on local and distant control in the brain were not provided. There was no radionecrosis reported in this group (56). Other reports on the outcome and safety of SRS for $\mathrm{BM}$ delivered after PCI include also the cases with prior WBRT for overt BM (57-62). In these series, median OS of patients after SRS ranged from 3 to 9 months. However, we should be aware that a selection bias in such retrospective series seriously impacts the results. Additionally, the local control after SRS for BM from SCLC was lower than for BM from other solid tumors. One-year local control rates were lower than $70 \%$ in evaluated patients (57-62), whilst in prospective trials on SRS with the exclusion of SCLC histology, these rates were of $70-90 \%(12,13)$. Distant brain control $(<60 \%)$ was also lower than that reported in prospective trials on SRS alone for non-SCLC histology (57-62). Nevertheless, the average patient with BM after PCI differs considerably from the patients participating in clinical trials on the use of ablative techniques for BM. For the former, very limited therapeutic options exist and his prognosis is ultimately fatal. If life expectancy exceeds 3 months and the technical possibilities for the use of SRS exist, we may proceed with SRS. WBRT at moderate doses is feasible for patients unsuitable for SRS or symptomatic patients with limited life expectancy ( $<3$ months) regardless of the technical possibilities of using SRS. For patients with poor performance status, we should consider supportive care only.

\section{SRS vs. WBRT for newly diagnosed BM in SCLC}

Whilst locally ablative treatments without WBRT are the standard of care for patients with 1-4 BM from solid tumors other than SCLC, the evidence for their use in SCLC is weak. The use of SRS for relapses after PCI or prior WBRT for overt BM in suitable cases is recommended, however, the upfront SRS for limited BM from SCLC is not a 
standard of care. The different biological behavior of SCLC prevented inclusion of these patients into most trials on local treatment of BM. The safety and potential benefit of such an approach remains to be confirmed in a prospective trial. However, the data on the futility of WBRT with respect to OS in BM from other solid tumors and the detrimental neurocognitive effect of WBRT, as well as the advancement in technologies and increasing availability of stereotactic techniques led to the growing use of upfront SRS for BM from SCLC. In one study, the availability of on-site SRS was the strongest factor related to the use of SRS for treatment of BM from solid tumors; $40 \%$ of patients who received WBRT had SRS when they were treated in a hospital that had on-site SRS technology, whilst only $3 \%$ of patients who received WBRT at a hospital without this technology had SRS, $\mathrm{P}<0.01$ (63). Indeed, one populationbased study demonstrated a positive trend in the use of SRS in 14,722 patients with BM from SCLC identified from the US NCDB covering the period of 2004-2013; the increase of the SRS use from $2.7 \%$ in 2004 to $4.3 \%$ in 2013 was observed. Although availability and socio-economic factors mainly influenced the use of SRS for these patients, the OS of patients receiving SRS was significantly superior to that of patients receiving WBRT only, the median OS rates were 10.0 vs. 8.0 vs. 9.3 for SRS, WBRT and WBRT plus SRS, respectively, $\mathrm{P}<0.001$. The OS differences favored the SRS group also following propensity score matching, $\mathrm{P}=0.001$ (7). Another study based on the US NCDB data included 5,952 BM SCLC patients and covered the period from 2010 to 2014. Upfront SRS was associated with superior OS than WBRT alone; median OS for 200 patients who had SRS was $10.8 v s$. 7.1 months for 5,752 WBRT patients, $\mathrm{P}<0.001$. These results were also confirmed in the propensity score matching analysis. Obviously, a small number of patients treated with SRS in these two studies, and a risk of undetected selection bias does not assert the value of the upfront SRS for these patients. Noteworthy, the OS of 7-8 months for patients in WBRT cohort was also higher than this reported in historical series (2-5). This may indicate that OS rates after SRS of about 3-9 months reported in case series would not be different if WBRT or other non-ablative radiotherapy techniques were used (56-62). However, the OS rates observed in the registry datasets suggest that upfront SRS may be appropriate for some SCLC patients. Patients with limited numbers of BM from SCLC may differ in their prognosis from patients with multiple BM. SCLC that occurs with single or oligo-BM may have more favorable prognosis than poly-metastatic brain disease at its onset. Thus, a more aggressive local approach would be beneficial for such patients. In the retrospective analysis of 52 patients who received WBRT for single BM SCLC, the use of surgery in combination with WBRT was related to improved survival compared with WBRT alone, with median OS of 19 and 5 months, respectively, $\mathrm{P}=0.03$ (64). On the prognostic scale for $\mathrm{BM}$ from lung cancer, based on the results of 1,833 NSCLC and 281 SCLC patients, the number of BM (1 vs. 2-3 vs. $>3$ ) reached prognostic significance. When patients with SCLC were analyzed separately, the number of BM was also significantly prognostic for survival and is included in the diagnosis-specific prognostic scale of BM from both NSCLC and SCLC (47).

The value of the upfront SRS, the safety of omission of WBRT for patients with a limited number of BM in SCLC should be evaluated in further prospective clinical trials. The ENCEPHALON trial registered in the clinicaltrials.gov website (NCT03297788) is recruiting a planned number of 56 patients at the Heidelberg University Hospital. Patients with up to $10 \mathrm{BM}$ from SCLC are randomized into the SRS of all lesions $v s$. WBRT. The primary endpoint of the study is neurocognitive function. The intracranial control, OS, quality of life and toxicity are secondary endpoints (65). More such studies are needed to accumulate the evidence regarding which patients with BM from SCLC can be safely managed with ablative techniques with omission of WBRT.

\section{Acknowledgments}

Funding: None.

\section{Footnote}

Provenance and Peer Review: This article was commissioned by the Guest Editor (Lucyna Kepka) for the series "Radiotherapy for Brain Metastases from Lung Cancer" published in Fournal of Thoracic Disease. The article has undergone external peer review.

Conflicts of Interest: All authors have completed the ICMJE uniform disclosure form (available at http://dx.doi. org/10.21037/jtd.2019.10.51). The series "Radiotherapy for Brain Metastases from Lung Cancer" was commissioned by the editorial office without any funding or sponsorship. LK served as the unpaid Guest Editor of the series and serves as an unpaid editorial board member of fournal of Thoracic Disease from Aug 2018 to Jul 2020. The other authors have 
no other conflicts of interest to declare.

Ethical Statement: The authors are accountable for all aspects of the work in ensuring that questions related to the accuracy or integrity of any part of the work are appropriately investigated and resolved.

Open Access Statement: This is an Open Access article distributed in accordance with the Creative Commons Attribution-NonCommercial-NoDerivs 4.0 International License (CC BY-NC-ND 4.0), which permits the noncommercial replication and distribution of the article with the strict proviso that no changes or edits are made and the original work is properly cited (including links to both the formal publication through the relevant DOI and the license). See: https://creativecommons.org/licenses/by-nc-nd/4.0/.

\section{References}

1. van Meerbeck JP, Fennell DA, De Ruysscher DK. Smallcell lung cancer. Lancet 2011; 378:1741-55.

2. Postmus PE, Haaxma-Reiche H, Gregor A, et al. Brainonly metastases of small cell lung cancer; efficacy of whole brain radiotherapy. An EORTC phase II study. Radiother Oncol 1998;46:29-32.

3. Postmus PE, Haaxma-Reiche H, Smit EF, et al. Treatment of brain metastases of small-cell lung cancer: comparing teniposide and teniposide with whole-brain radiotherapy-a phase III study of the European Organization for the Research and Treatment of Cancer Lung Cancer Cooperative Group. J Clin Oncol 2000;18:3400-8.

4. Kepka L, Cieslak E, Bujko K, et al. Results of the wholebrain radiotherapy for patients with brain metastases from lung cancer: the RTOG RPA intra-classes analysis. Acta Oncol 2005;44:389-98.

5. Videtic GM, Adelstein DJ, Mekhail TM, et al. Validation of the RTOG recursive partitioning analysis (RPA) classification for small-cell lung cancer-only brain metastases. Int J Radiat Oncol Biol Phys 2007;67:240-3.

6. Lally BE, Geiger AM, Urbanic JJ, et al. Trends in the outcomes for patients with limited stage small cell lung cancer: an analysis of the surveillance, epidemiology, and end results database. Lung Cancer 2009;64:226-31.

7. Jiang $W$, Haque $W$, Verma $V$, et al. Stereotactic radiosurgery for brain metastases from newly diagnosed small cell lung cancer: practice patterns and outcomes. Acta Oncol 2019;58:491-8.

8. Robin TP, Jones BL, Amini A, et al. Radiosurgery alone is associated with favorable outcomes for brain metastases from small-cell lung cancer. Lung Cancer 2018;120:88-90.

9. Aupérin A, Arriagada R, Pignon JP, et al. Prophylactic cranial irradiation for patients with small cell lung cancer in complete remission. Prophylactic Cranial Irradiation Overview Collaborative Group. N Engl J Med 1999;341:476-84.

10. Slotman B, Faivre-Finn C, Kramer G, et al. Prophylactic cranial irradiation in extensive small-cell lung cancer. $\mathrm{N}$ Engl J Med 2007;357:664-72.

11. Andrews DW, Scott CB, Sperduto PW, et al. Whole brain radiation therapy with or without stereotactic radiosurgery boost for patients with one to three brain metastases: phase III results of the RTOG 9508 randomised trial. Lancet 2004;363:1665-72.

12. Aoyama H, Shirato H, Tago M, et al. Stereotactic radiosurgery plus whole-brain radiation therapy vs stereotactic radiosurgery alone for treatment of brain metastases: a randomized controlled trial. JAMA 2006;295:2483-91.

13. Kocher M, Soffietti R, Abacioglu U, et al. Adjuvant wholebrain radiotherapy versus observation after radiosurgery or surgical resection of one to three cerebral metastases: results of the EORTC 22952-26001 study. J Clin Oncol 2011;29:134-41.

14. Brown PD, Ballman KV, Cerhan JH, et al. Postoperative stereotactic radiosurgery compared with whole brain radiotherapy for resected metastatic brain disease (NCCTG N107C/CEC.3): a multicentre, randomised, controlled, phase 3 trial. Lancet Oncol 2017;18:1049-60.

15. Kępka L, Tyc-Szczepaniak D, Bujko K, et al. Stereotactic radiotherapy of the tumor bed compared to whole brain radiotherapy after surgery of single brain metastasis: results from a randomized trial. Radiother Oncol 2016;121:217-24.

16. Jett JR, Schild SE, Kesler KA, et al. Treatment of small cell lung cancer: Diagnosis and management of lung cancer, 3rd ed: American College of Chest Physicians evidence based clinical practice guidelines. Chest 2013;143:e400S-19S.

17. Takahashi T, Yamanaka T, Seto T, et al. Prophylactic cranial irradiation versus observation in patients with extensive-disease small-cell lung cancer: a multicentre, randomised, open-label, phase 3 trial. Lancet Oncol 2017;18:663-71.

18. Ginsberg S, Kirshner J, Reich S, et al. Systemic chemotherapy for a primary germ cell tumor of the brain: a pharmacokinetic study. Cancer Treat Rep 1981;65:477-83.

19. Grossi F, Scolaro T, Tixi L, et al. The role of systemic chemotherapy in the treatment of brain metastases 
from small-cell lung cancer. Crit Rev Oncol Hematol 2001;37:61-7.

20. Seute T, Leffers P, Wilmink JT, et al. Response of asymptomatic brain metastases from small-cell lung cancer to systemic first-line chemotherapy. J Clin Oncol 2006;24:2079-83.

21. Reveiz L, Rueda JR, Cardona AF. Chemotherapy for brain metastases from small cell lung cancer. Cochrane Database Syst Rev 2012;(6):CD007464.

22. Horn L, Mansfield AS, Szczesna A, et al. First-line Atezolizumab plus chemotherapy in Extensive-Stage Small-Cell Lung Cancer. N Engl J Med 2018;379:2220-9.

23. Renz P, Hasan S, Wegner RE. Survival outcomes after whole brain radiotherapy for brain metastases in older adults with newly diagnosed metastatic small cell carcinoma: A national cancer database (NCDB) analysis. J Geriatr Oncol 2019;10:560-6.

24. Zhang W, Jiang W, Luan L, et al. Prophylactic cranial irradiation for patients with small cell lung cancer: a systematic review of the literature with meta-analysis. BMC Cancer 2014:14:793.

25. Socha J, Kepka L. Prophylactic cranial irradiation for small cell lung cancer: how, when and for whom? Expert Rev Anticancer Ther 2012;12:505-17.

26. Le Péchoux C, Dunant A, Senan S, et al. Standard-dose versus higher-dose prophylactic cranial irradiation (PCI) in patients with limited-stage small-cell lung cancer in complete remission after chemotherapy and thoracic radiotherapy (PCI 99-01, EORTC 22003-08004, RTOG 0212, and IFCT 99-01): a randomised clinical trial. Lancet Oncol 2009;10:467-74.

27. Wolfson AH, Bae K, Komaki R, et al. Primary analysis of a phase II randomized trial Radiation Therapy Oncology Group (RTOG) 0212: impact of different total doses and schedules of prophylactic cranial irradiation on chronic neurotoxicity and quality of life for patients with limiteddisease small-cell lung cancer. Int J Radiat Oncol Biol Phys 2011;81:77-84.

28. National Comprehensive Cancer Network Clinical Practice Guidelines in Oncology. Small Cell Lung Cancer (Version 1.2019). Available online: www.nccn.org/ professionals/physician_gls/pdf/sclc.pdf

29. Davey P, Ennis M, Aviv R. Prophylactic cranial irradiation (PCI). Still a no-brainer? Lung Cancer 2015;89:4-7.

30. Rusthoven CG, Kavanagh BD. Prophylactic Cranial Irradiation (PCI) versus Active MRI Surveillance for Small Cell Lung Cancer: The Case for Equipoise. J. Thorac Oncol 2017;12:1746-54.

31. Farrell MJ, Yahya JB, Degnin C, et al. Prophylactic cranial irradiation for limited-stage small cell lung cancer: A survey of US radiation oncologists on current practice patterns. Clin Lung Cancer 2019;20:13-9.

32. Péchoux CL, Sun A, Slotman BJ, et al. Prophylactic cranial irradiation for patients with lung cancer. Lancet Oncol 2016;17:e277-93.

33. Putora PM, Glatzer M, Belderbos J et al. Prophylactic cranial irradiation in stage IV small cell lung cancer: Selection of patients amongst European IASLC and ESTRO experts. Radiother Oncol 2019;133:163-6.

34. Manapov F, Klautke G, Fietkau R. Prevalence of brain metastases immediately before prophylactic cranial irradiation in limited disease small cell lung cancer patients with complete remission to chemoradiotherapy: a single institution experience. J Thorac Oncol 2008;3:652-5.

35. Chu X, Yang X, Zhu Z. Prevalence and risk factors of brain metastases in limited stage small cell lung cancer immediately before prophylactic cranial irradiation. J Thorac Oncol 2018;10:S436.

36. Suwinski R, Lee SP, Withers HR. Dose-response relationship for prophylactic cranial irradiation in small cell lung cancer. Int J Radiat Oncol Biol Phys 1998;40:797-806.

37. Gondi V, Pugh SL, Tome WA, et al. Preservation of memory with conformal avoidance of the hippocampal neural stem-cell compartment during whole-brain radiotherapy for brain metastases (RTOG0933): a phase II multi-institutional trial. J Clin Oncol 2014;32:3810-6.

38. Gondi V, Deshmukh S, Brown PD, et al. Preservation of neurocognitive function (NCF) with conformal avoidance of the hippocampus during whole-brain radiotherapy (HAWBRT) for brain metastases: preliminary results of phase III trial NRG Oncology CC001. Int J Radiat Oncol Biol Phys 2018;102:1607.

39. Kundapur V, Ellchuk T, Ahmed S, Gondi V. Risk of hippocampal metastases in small cell lung cancer patients at presentation and after cranial irradiation: a safety profile study for hippocampal sparing during prophylactic or therapeutic cranial irradiation. Int J Radiat Oncol Biol Phys 2015;91:781-6.

40. Harth S, Abo-Madyan Y, Zheng L, et al. Estimation of intracranial failure risk following hippocampal-sparing whole brain radiotherapy. Radiother Oncol 2013;109:152-8.

41. Redmond KJ, Hales RK, Anderson-Keightly H, et al. Prospective Study of Hippocampal-Sparing Prophylactic Cranial Irradiation in Limited-Stage Small Cell Lung Cancer. Int J Radiat Oncol Biol Phys 2017;98:603-11.

42. Gondi V, Tome WA, Rowley HA, Mehta MP. Hippocampal Contouring: A contouring atlas for RTOG 
0933. Available online: https://www.rtog.org/CoreLab/ ContouringAtlases/HippocampalSparing.aspx

43. Bernhardt D, Adeberg S, Bozorgmehr F, et al. Outcome and prognostic factors in patients with brain metastases from small-cell lung cancer treated with whole brain radiotherapy. J Neurooncol 2017;134:205-12.

44. Bernhardt D, König L, Aufderstrasse S, et al. Generation of a new disease-specific prognostic score for patients with brain metastases from small-cell lung cancer treated with whole brain radiotherapy (BMS-Score) and validation of two other indices. Clin Lung Cancer 2018;19:340-5.

45. Puglisi M, Dolly S, Faria A, et al. Treatment options for small cell lung cancer-do we have more choice? Br J Cancer 2010;102:629-38.

46. Gaspar L, Scott C, Rotman M, et al. Recursive partitioning analysis (RPA) of prognostic factors in three Radiation Therapy Oncology Group (RTOG) brain metastases trials. Int J Radiat Oncol Biol Phys 1997;37:745-51.

47. Sperduto PW, Kased N, Roberge D, et al. Summary report on the graded prognostic assessment: an accurate and facile diagnosis-specific tool to estimate survival for patients with brain metastases. J Clin Oncol 2012;30:419-25.

48. Neuhaus T, Ko Y, Muller RP, et al. A phase III trial of topotecan and whole brain radiation therapy for patients with CNS-metastases due to lung cancer. British Journal of Cancer 2009;100:291-7.

49. Liu Y, Liu XH, Wang Y, et al. A study on different therapies and prognosis-related factors for 101 patients with SCLC and brain metastases. Cancer Biol Ther 2017;18:670-5.

50. Liu M, Zhou Y, Han Q, et al. Whole brain radiotherapy concomitant or sequential Vm26/DDP in treating small cell lung cancer patients with brain metastases. Chin-Ger J Clin Oncol 2010;9:17-21.

51. Mulvenna P, Nankivell M, Barton R, et al. Dexamethasone and supportive care with or without whole brain radiotherapy in treating patients with non-small cell lung cancer with brain metastases unsuitable for resection or stereotactic radiotherapy (QUARTZ): results from a phase 3, noninferiority, randomised trial. Lancet 2016;388:2004-14.

52. Komosinska K, Kepka L, Niwinska A, et al. Prospective evaluation of the palliative effect of whole-brain radiotherapy in patients with brain metastases and poor performance status. Acta Oncol 2010;49:382-8.

53. Lawrence YR, Li XA, el Naqa I, et al. Radiation dosevolume effects in the brain. Int J Radiat Oncol Biol Phys 2010;76:S20-7.

54. Yamamoto M, Serizawa T, Shuto T, et al. Stereotactic radiosurgery for patients with multiple brain metastases (JLGK0901): a multi-institutional prospective observational study. Lancet Oncol 2014;15:387-95.
55. Mazure B, Guest N, Letcher A, et al. Should stereotactic radiosurgery be considered for salvage of intracranial recurrence in small cell lung cancer? J Thorac Oncol 2018;13:S436.

56. Bernhardt D, Bozorgmehr F, Adeberg S, et al. Outcome in patients with small cell lung cancer re-irradiated for brain metastases after prior prophylactic cranial irradiation. Lung Cancer 2016;101:76-81.

57. Harris S, Chan MD, Lovato JF, et al. Gamma knife stereotactic radiosurgery as salvage therapy after failure of whole-brain radiotherapy in patients with small cell lung cancer. Int J Radiat Oncol Biol Phys 2012;83:e53-9.

58. Rava P, Sioshansi S, DiPetrillo T, et al. Local recurrence and survival following stereotactic radiosurgery for brain metastases from small cell lung cancer. Pract Radiat Oncol 2015;5:e37-44.

59. Nakazaki K, Higuchi Y, Nagano O, et al. Efficacy and limitations of salvage gamma knife radiosurgery for brain metastases of small-cell lung cancer after wholebrain radiotherapy. Acta Neurochir 2013;155:107-13; discussion 113-4.

60. Olson AC, Wegner RE, Rwigema JCM, et al. Clinical outcomes of reirradiation of brain metastases from small cell lung cancer with cyberknife stereotactic radiosurgery. J Cancer Res Ther 2012;8:411-6.

61. Wegner RE, Olson AC, Kondziolka D, et al. Stereotactic radiosurgery for patients with brain metastases from small cell lung cancer. Int J Radiat Oncol Biol Phys 2011;81:e21-7.

62. Yomo S, Hayashi M. Is stereotactic radiosurgery a rational treatment option for brain metastases from small cell lung cancer? A retrospective analysis of 70 consecutive patients. BMC Cancer 2015;15:95.

63. Hodgson DC, Charpentier AM, Cigsar C, et al. A multiinstitutional study of factors influencing the use of stereotactic radiosurgery for brain metastases. Int J Radiat Oncol Biol Phys 2013;85:335-40.

64. Bernhardt D, Adeberg S, Bozorgmehr F, et al. Outcome and prognostic factors in single brain metastases from small-cell lung cancer. Strahlenther Onkol 2018;194:98-106.

65. Bernhardt D, Hommertgen A, Schmitt D, et al. Whole brain radiation therapy alone versus radiosurgery for patients with 1-10 brain metastases from small cell lung cancer (ENCEPHALON Trial): study protocol for a randomized controlled trial. Trials 2018;19:388.

Cite this article as: Kepka L, Socha J, Sas-Korczynska B. Radiotherapy for brain metastases from small-cell lung cancer in distinct clinical indications and scenarios. J Thorac Dis 2021;13(5):3269-3278. doi: 10.21037/jtd.2019.10.51 